TI 2011-182/1

Tinbergen Institute Discussion Paper

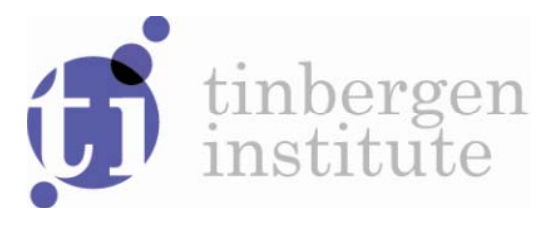

\title{
Harsanyi Power Solutions for Games on Union Stable Systems
}

Encarnacion Algabal

lesus Mario Bilbaol

René van den Brink²

1 University of Seville;

2 Faculty of Economics and Business Administration, VU University Amsterdam, and Tinbergen Institute. 
Tinbergen Institute is the graduate school and research institute in economics of Erasmus University Rotterdam, the University of Amsterdam and VU University Amsterdam.

More TI discussion papers can be downloaded at http://www.tinbergen.nl

Tinbergen Institute has two locations:

Tinbergen Institute Amsterdam

Gustav Mahlerplein 117

1082 MS Amsterdam

The Netherlands

Tel.: +31(0)205251600

Tinbergen Institute Rotterdam

Burg. Oudlaan 50

3062 PA Rotterdam

The Netherlands

Tel.: +31(0)10 4088900

Fax: $+31(0) 104089031$

Duisenberg school of finance is a collaboration of the Dutch financial sector and universities, with the ambition to support innovative research and offer top quality academic education in core areas of finance.

DSF research papers can be downloaded at: http://www.dsf.nl/

Duisenberg school of finance

Gustav Mahlerplein 117

1082 MS Amsterdam

The Netherlands

Tel.: +31(0)20 5258579 


\title{
Harsanyi power solutions for games on union stable systems
}

\author{
E. Algaba*, J. M. Bilbao*, R. van den Brink** \\ *Matemática Aplicada II, Escuela Superior de Ingenieros, Camino de los \\ Descubrimientos, s/n, 41092 Sevilla, Spain (E-mail: ealgaba@us.es) \\ ** Department of Econometrics and Tinbergen Institute, VU University, De \\ Boelelaan 1105, 1081 HV Amsterdam, The Netherlands (E-mail: \\ jbrink@feweb.vu.nl)
}

\begin{abstract}
This paper analyzes Harsanyi power solutions for cooperative games in which partial cooperation is based on union stable systems. These structures contain as particular cases the widely studied communication graph games and permission structures, among others. In this context, we provide axiomatic characterizations of the Harsanyi power solutions which distribute the Harsanyi dividends proportional to weights determined by a power measure for union stable systems. Moreover, the well-known Myerson value is exactly the Harsanyi power solution for the equal power measure, and on a special subclass of union stable systems the position value coincides with the Harsanyi power solution obtained for the influence power measure.
\end{abstract}

\section{JEL Classification C71}

Key words: Cooperative TU-game, Union stable system, Harsanyi dividend, Power measure, Harsanyi power solution, Myerson value, Position value.

\section{Introduction}

In the classical model of cooperative games with transferable utility it is generally assumed that there are no restrictions on cooperation. However, in practice, many situations require certain limitations on cooperation. Myerson [20] used ideas from graph theory and studied how the outcome of a game depends on which players cooperate with each other. This line of research was then continued by Owen [24], Borm et al. [6], and Potters and Reijnierse [25]. However, Myerson himself pointed out that partial cooperation can not always be modelled by a graph. Therefore, Myerson's communication model has been generalized in several directions, for instance towards conference structures by Myerson [21], hypergraph communication situations by van den Nouweland et al. [23] and union stable systems by Algaba et al. [1, 2]. In this paper we consider Harsanyi power solutions for union stable structures, generalizing results of van den Brink et al. [9] who introduce this class of 
solutions for communication graph games, and results on the Myerson and position value for union stable structures in Algaba et al. ([1],[2],[4]).

The union stable system model introduced by Algaba et al. [1,2] assumes that if two feasible coalitions have common elements, these will act as intermediaries between the two coalitions in order to establish meaningful cooperation in the union of these coalitions. In other words, players in feasible coalitions can communicate, so players in the intersection of two feasible coalitions make communication in the union coalition possible. This mathematical feature is relevant, and feasible coalition systems coming from communication graphs $([20])$, permission structures $([7,14])$, systems under precedence constraints ([15]), antimatroids ([3]) and augmenting systems ([5]) verify this condition, thus being special cases of union stable systems. Furthermore, these systems have a close relation with the hypergraph communication situations. Recently, Faigle et al. [16] have established union stable systems as the more general systems where it is possible to define a meaningful notion of supermodularity that generalizes Shapley's original convexity model for classical cooperative games.

To illustrate the importance of union stable systems, consider a network of people that are in the Board of Directors of big companies. Typically, such people are members of the board of several companies, see e.g., Mizruchi and Bunting [19] and Conyon and Muldoon [11]. Besides the influence that a board member has on a company, it is interesting to know what influence board members have on each other when they are member of the board of the same company. Or, even more interesting, what is the influence of a board member of company A on a board member of company B, while they are in no board together, but have a third board member who is sharing a board with each of them. And even there can be influence without such a third member, but more indirect relations. In general, a Board of Directors of a company consists of more than two members, and thus the network where the basis elements are the sets of people that belong to the board of one company cannot be modelled by a simple graph. However, we can consider the boards as the basis of a union stable system.

The aim of this paper is to study Harsanyi power solutions introduced by van den Brink et al. [9] for communication graph games, in the context where cooperation is based on union stable cooperation structures. Harsanyi solutions are introduced and studied as solutions for TU-games in Vasil'ev ([27], [28]) and Derks et al. [12, 13]. In our context, the Harsanyi dividends ([17]) in the cooperation restricted game are distributed according to a sharing system determined by a positive power measure defined for union stable systems. Such a power measure assigns a non-negative real number to every player which measures the power or strength of this player in the union stable system which is considered. Given a power measure, the corresponding sharing system is defined in such way that the share vectors for any coalition $S$ are proportional to the power measure of the union stable subsystem obtained for those feasible coalitions contained in $S$. Special positive power 
measures for union stable systems are the influence measure and the equal power measure which generalizes the degree and equal power measures for the particular case of communication graphs. We analyze these Harsanyi power solutions, establishing that for specific power measures they coincide with some well-known solutions in the literature. For example, on a special class of union stable systems, denoted by $U S I^{N}$ and defined as those that are closed under intersection (if the intersection contains at least two elements) and such that every non-unitary feasible coalition can be written in a unique way as a union of non-unitary supports (this contains the class of cycle-free communication graph situations), the Harsanyi power solution obtained for the influence measure is equal to the position value as defined in [1] and on the union stable family $2^{N}$ coincides with the Shapley value. In addition to this, it is interesting to notice that on the class of all union stable structures the Myerson value ([2]) coincides with the Harsanyi solution obtained for the equal power measure.

Generalizing the approach of van den Brink et al. [9], we provide a unified approach by axiomatizing the Harsanyi power solutions with the same axioms except one which states that for special games on union stable systems, we allocate the earnings proportional to some power measure for union stable systems. This shows that the essential difference will be in the measure considered. Concretly, on the class $U S I^{N}$ we can extend the argument in [9] that the fundamental difference between the Myerson value and the position value is with respect to the power measure that is applied (the equal power measure or the influence power measure) to union stable structures. In fact, on the subclass $U S I^{N}$ we obtain two axiomatic characterizations for the Harsanyi power solutions using on the one hand $\sigma$-point unanimity and on the other hand the $\sigma$-influence property which, when taking the same power measure, are equivalent on the class $U S I^{N}$. On the class of all union stable structures, $\sigma$-point unanimity implies the $\sigma$-influence property. Applying the equal power measure and the influence power measure we obtain new characterizations for the Myerson and position value for union stable structures, respectively.

Another axiom that we use in the axiomatizations on $U S I^{N}$ is the superfluous support property. Although the Myerson value satisfies the superfluous support property on the class of all union stable structures, this is not the case for all Harsanyi power solutions. We show that replacing the superfluous support property by either the superfluous player property of Algaba et al. [1] (generalizing the superfluous player property for communication graph games of van den Nouweland [23]) or generalizing the inessential link property and connectedness of van den Brink et al. [9], leads to characterizations on the class of all union stable structures.

The paper is organized as follows. Section 2 recalls the main concepts on cooperative TU-games and union stable systems which will be used in the following sections. In Section 3, we introduce the notion of Harsanyi power solutions for union stable systems. In Section 4, we analyze some of 
their properties. In Section 5, we provide axiomatic characterizations of the Harsanyi power solutions on the class $U S I^{N}$ that generalizes the cycle-free communication situations, using the superfluous support property. In Section 6 , we provide axiomatizations on the class of all games on union stable systems by using either the superfluous player property or the inessential support property and connectedness which gives rise to a new axiomatization of the Myerson value on the class of all union stable structures. Finally, in Section 7 we give some special attention to the Myerson value

\section{Preliminaries}

\subsection{Cooperative TU-games}

A cooperative transferable utility (TU)-game is a pair $(N, v)$ where $N=$ $\{1, \ldots, n\}$ is a finite set of players and $v: 2^{N} \rightarrow \mathbb{R}$ with $v(\emptyset)=0$, is a characteristic function.

A distribution of the amount $v(N)$ among the players will be represented by a real-valued vector $x \in \mathbb{R}^{n}$. Here $x_{i}$ represents the payoff to player $i$ according to the involved payoff vector $x$. A solution is a real-valued function that assigns a payoff vector to every game $(N, v)$. A solution $f$ satisfies the efficiency principle if $\sum_{j \in N} f_{j}(N, v)=v(N)$.

The most well-known solution is the Shapley value (Shapley [26]) given by

$$
\Phi_{i}(N, v)=\sum_{S \subseteq N \backslash\{i\}} \frac{(n-|S|-1) !(|S|) !}{n !}(v(S \cup\{i\})-v(S)) \text {, for all } i \in N \text {. }
$$

For each $T \subseteq N$, the unanimity game $u_{T}$ is given by $u_{T}(S)=1$ if $T \subseteq$ $S$, and $u_{T}(S)=0$ otherwise. It is well-known that the unanimity games $u_{T}, T \subseteq N, T \neq \emptyset$, form a basis of the vectorial space of TU-games on $N$ denoted by $\mathcal{G}^{N}$, and that each game $v \in \mathcal{G}^{N}$ can be written as a linear combination of unanimity games in a unique way: $v=\sum_{T \subseteq N, T \neq \emptyset} \Delta_{v}(T) u_{T}$, where the coefficients $\Delta_{v}(T)$ are the Harsanyi dividends, (see Harsanyi [17]). By applying the Möbius transformation we obtain that

$$
\Delta_{v}(S)=\sum_{T \subseteq S}(-1)^{|S|-|T|} v(T), \quad S \subseteq N .
$$

The Shapley value belongs to the class of Harsanyi solutions, proposed by Vasil'ev ([27], [28]), also known as sharing values, see Derks et al. [12]. First, a sharing system on $N$ is a system $p=\left(p^{S}\right)_{S \subseteq N, S \neq \emptyset}$, where $p^{S}$ is an $|S|$-dimensional vector assigning a nonnegative share $p_{i}^{S} \geq 0$ to every player $i \in S$ with $\sum_{j \in S} p_{j}^{S}=1, S \subseteq N, S \neq \emptyset$. We denote the collection of all sharing systems on $N$ by $P^{N}$. For a game $(N, v)$ and sharing system $p \in P^{N}$, the corresponding Harsanyi payoff vector is the payoff vector $h^{p}(N, v) \in \mathbb{R}^{n}$ given by

$$
h_{i}^{p}(N, v)=\sum_{S \subseteq N, i \in S} p_{i}^{S} \Delta_{v}(S), \quad i \in N,
$$


i.e., the payoff $h_{i}^{p}(N, v)$ to player $i \in N$ is the sum over all coalitions $S \subseteq N$, containing $i$, of the share $p_{i}^{S} \Delta_{v}(S)$ of player $i$ in the Harsanyi dividend of coalition $S$. A Harsanyi solution on $\mathcal{G}^{N}$ assigns to a given sharing system $p \in$ $P^{N}$ the Harsanyi payoff vector $h^{p}(N, v)$ to each game $(N, v)$. By definition, the Harsanyi solutions are efficient. The Shapley value is the Harsanyi solution that assigns to any game $(N, v)$ the Harsanyi payoff vector $h^{p}(N, v)$ with the sharing system $p$ given by $p_{i}^{S}=\frac{1}{|S|}, S \subseteq N, i \in S$.

\subsection{Union stable systems and structures}

Let $N=\{1, \ldots, n\}$ be a finite set of players and $\mathcal{F} \subseteq 2^{N}$ a set system of feasible coalitions. Union stable systems are introduced in Algaba et al. [1]. Formally, the set system $\mathcal{F}$ is called union stable if for all $A, B \in \mathcal{F}$ with $A \cap B \neq \emptyset$ it is satisfied that $A \cup B \in \mathcal{F}$.

Many real world situations find its natural framework in these structures. For instance, suppose that player 1 is a homeowner who wants to sell his/her house. Player 1 has signed a contract with a real estate agent that represents player 2. So, player 1 only can sell his/her house by means of player 2 . There are two buyers, players 3 and 4 . Notice that in this economic application, the family of feasible coalitions that can generate a surplus are only those which make possible that the seller can sell his/her house. Therefore, the coalitions which can trade are

$$
\mathcal{F}=\{\{1,2,3\},\{1,2,4\},\{1,2,3,4\}\} .
$$

An important subclass of union stable systems are communication graphs as considered in Myerson [20]. A communication graph game is a triple $(N, v, E)$, where $(N, v)$ is a game and $(N, E)$ is a simple graph. It is easy to see that the set system $\mathcal{F}$, defined by those coalitions which induce connected subgraphs, is a union stable system. However, in practice, a union stable system can not always be modelled by a communication graph (see van den Brink [8] for a characterization of the set systems that can be obtained as connected coalitions in a communication graph). For example, the set system $\mathcal{F}$ pointed out above with one seller/two buyers and a real state agent as intermediary, is a union stable system which cannot be the set of connected coalitions in a communication graph. So, union stable systems not only allow for a generalization of set systems derived from communication graphs but also a better understanding and insight of them and their applications.

Let $\mathcal{F}$ be a union stable system and $\mathcal{G} \subseteq \mathcal{F}$. In order to obtain the basis of a union stable system (see [1]), the following families are defined inductively

$$
\mathcal{G}^{(0)}=\mathcal{G}, \quad \mathcal{G}^{(n)}=\left\{S \cup T: S, T \in \mathcal{G}^{(n-1)}, S \cap T \neq \emptyset\right\}, \quad(n=1,2, \ldots) .
$$

Notice that $\mathcal{G}^{(0)} \subseteq \mathcal{G}^{(n-1)} \subseteq \mathcal{G}^{(n)} \subseteq \mathcal{F}$, since $\mathcal{G} \subseteq \mathcal{F}$ and $\mathcal{F}$ is union stable. We define $\overline{\mathcal{G}}$ by $\overline{\mathcal{G}}=\mathcal{G}^{(\bar{k})}$, where $k$ is the smallest integer such that $\mathcal{G}^{(k+1)}=\mathcal{G}^{(k)}$. 
For each union stable family $\mathcal{F}$, it is interesting to find a minimal subset $\mathcal{B}(\mathcal{F})$ such that $\overline{B(\mathcal{F})}=\mathcal{F}$. So, the following set is well-defined:

$$
\mathcal{E}(\mathcal{F})=\{G \in \mathcal{F}: G=A \cup B, A \neq G, B \neq G, A, B \in \mathcal{F}, A \cap B \neq \emptyset\} .
$$

The set $\mathcal{B}(\mathcal{F})=\mathcal{F} \backslash \mathcal{E}(\mathcal{F})$, is called the basis of $\mathcal{F}$, and the elements of $\mathcal{B}(\mathcal{F})$ are called supports of $\mathcal{F}$. We remark that the basis $B(\mathcal{F})$ is the minimal subset of the union stable system $\mathcal{F}$ such that $\overline{B(\mathcal{F})}=\mathcal{F}$ (see Algaba et al. $[1])$.

Let $\mathcal{G} \subseteq 2^{N}$ be a set system and let $S \subseteq N$. A set $T \subseteq S$ is called a $\mathcal{G}$-component of $S$ if $T \in \mathcal{G}$ and there exists no $T^{\prime} \in \mathcal{G}$ such that $T \subset T^{\prime} \subseteq S$. Therefore, the $\mathcal{G}$-components of $S$ are the maximal feasible coalitions that belong to $\mathcal{G}$ and are contained in $S$. We denote by $C_{\mathcal{G}}(S)$ the collection of the $\mathcal{G}$-components of $S$. Union stable systems can be characterized in terms of the $\mathcal{F}$-components of a coalition in the following way: The set system $\mathcal{F} \subseteq 2^{N}$ is union stable if and only if for any $S \subseteq N$ with $C_{\mathcal{F}}(S) \neq \emptyset$, the $\mathcal{F}$-components of $S$ are a collection of pairwise disjoint subsets of $S$, (see [1]). So, if for every $i \in N$, there is an $S \in \mathcal{F}$ such that $i \in S$ then the $\mathcal{F}$-components form a partition of the player set $N$.

Let $(N, v)$ be a cooperative game and $\mathcal{F} \subseteq 2^{N}$ a union stable system. Let $\mathcal{B}(\mathcal{F})$ be the basis of $\mathcal{F}$ and $\mathcal{C}(\mathcal{F})=\{B \in \mathcal{B}(\mathcal{F}):|B| \geq 2\}$. If there is no confusion we will just write $\mathcal{B}$ and $\mathcal{C}$. The $\mathcal{F}$-restricted game $v^{\mathcal{F}}: 2^{N} \rightarrow \mathbb{R}$, is defined on the player set $N$ and is given by $v^{\mathcal{F}}(S)=\sum_{T \in C_{\mathcal{F}}(S)} v(T)$. On the other hand, the conference game is defined on the basis of a union stable system $^{1}$ (so, on player set $\mathcal{C}$ ) and it is the game $\left(\mathcal{C}, v^{\mathcal{C}}\right)$ where $v^{\mathcal{C}}: 2^{\mathcal{C}} \rightarrow \mathbb{R}$, is given by $v^{\mathcal{C}}(\mathcal{A})=v^{\overline{\mathcal{A}}}(N)$.

Note that the game $\left(\mathcal{C}, v^{\mathcal{C}}\right)$ is well defined since for each $\mathcal{A} \subseteq \mathcal{C}, \overline{\mathcal{A}}$ is a union stable system. The $\mathcal{F}$-restricted game focuses on the role of a player in creating economic possibilities and establishing meaningful communication among the players, whereas the conference game measures the economic value of the grand coalition when specific parts of the cooperation structure are considered.

The two above definitions extend the point game (introduced by Myerson [20]) and the arc game (introduced by Borm et al. [6]) for communication graph games, where for a communication graph game $(N, v, E)$ we have that $\mathcal{C}=\{\{i, j\}:\{i, j\} \in E\}$.

A union stable cooperation structure is a triple $(N, v, \mathcal{F})$ where $N=$ $\{1, \ldots, n\}$ is the set of players, $(N, v)$ is a game with $v: 2^{N} \rightarrow \mathbb{R}$ satisfying $v(\emptyset)=0$, and $\mathcal{F}$ is a union stable system. For convenience, we assume from now on that the underlying game $(N, v)$ is zero-normalized, i.e., $v(\{i\})=0$ for all $i \in N$.

\footnotetext{
${ }^{1}$ Although in the beginning of this section we mentioned that we always take as player set $N=\{1, \ldots, n\}$, the definition of the conference game is the only occasion where we deviate from this. Note that the player set in a conference game is still derived from a structure on $N$.
} 
The set of all union stable cooperation structures on $N$ will be denoted by $U S^{N}=\{(N, v, \mathcal{F}): \mathcal{F}$ is union stable $\}$.

We denote by $U S I^{N}$ the special subclass of $U S^{N}$ where the following two conditions are satisfied:

(1) For all $S, T \in \mathcal{F}$ with $|S \cap T| \geq 2$ we have $S \cap T \in \mathcal{F}$.

(2) All non-unitary feasible coalitions can be written in a unique way as a union of non-unitary supports.

Notice that this subclass of union stable cooperation structures generalizes those communication graph games for which the graphs do not contain cycles.

\subsection{Allocation rules for union stable cooperation structures}

An allocation rule on $U S^{N}$ is a map $\gamma$ that assigns to each union stable cooperation structure $(N, v, \mathcal{F})$ a payoff vector, $\gamma(N, v, \mathcal{F}) \in \mathbb{R}^{n}$.

Both the Myerson value and the position value are defined from the Shapley value $[26]$, but using the $\mathcal{F}$-restricted game and the conference game, respectively, which were defined in the previous subsection. The Myerson value was introduced in Myerson [20] and later extended in [21]. Myerson pointed out the need to generalize this model towards restricted cooperation situations which can not be modelled by a graph. This idea has been studied by van den Nouweland et al. [23] and Algaba et al. [2]. Given a union stable cooperation structure $(N, v, \mathcal{F})$, the Myerson value, denoted by $\mu(N, v, \mathcal{F}) \in \mathbb{R}^{n}$, is defined by

$$
\mu(N, v, \mathcal{F})=\Phi\left(N, v^{\mathcal{F}}\right) .
$$

The position value for communication graph situations was first introduced in Meesen [18] and studied in Borm et al. [6]. This value was extended to hypergraph communication situations in [23] and it is defined on union stable cooperation structures in [1]. Let $(N, v, \mathcal{F})$ be a union stable cooperation structure. The position value, denoted by $\pi(N, v, \mathcal{F}) \in \mathbb{R}^{n}$, is given by

$$
\pi_{i}(N, v, \mathcal{F})=\sum_{C \in \mathcal{C}_{i}(\mathcal{F})} \frac{1}{|C|} \Phi_{C}\left(\mathcal{C}, v^{\mathcal{C}}\right), \text { for } i \in N
$$

where $\mathcal{C}_{i}(\mathcal{F})=\{C \in \mathcal{C}: i \in C\}$. When there is no confusion we will often write $\mathcal{C}_{i}$ instead of $\mathcal{C}_{i}(\mathcal{F})$.

\section{Harsanyi power solutions for union stable cooperation struc- tures}

In van den Brink et al. [9] the class of Harsanyi power solutions for communication graph games is introduced. A main purpose of the underlying paper is to extend these solutions to the union stable cooperation structures since above we illustrated that many networks in society cannot be modelled by a simple graph. 
We denote the class of all union stable systems on $N$ by $\mathcal{U}^{N}$. A power measure for union stable systems is a function $\sigma: \mathcal{U}^{N} \rightarrow \mathbb{R}_{+}^{n}$ that assigns to any union stable system $\mathcal{F}$, a nonnegative vector $\sigma(N, \mathcal{F}) \in \mathbb{R}_{+}^{n}$, yielding the nonnegative power $\sigma_{i}(N, \mathcal{F})$ of player $i \in S$ in the union stable system $\mathcal{F}$. A power measure is symmetric if for any union stable system $\mathcal{F}$ and $i, j \in N$, $S \in \mathcal{F}$ such that $i \in S$ if and only if $j \in S$ we have $\sigma_{i}(N, \mathcal{F})=\sigma_{j}(N, \mathcal{F})$. It is positive if for any $\mathcal{F} \in \mathcal{U}^{N}$, the power of player $i$ is positive if and only if $i \in M$ for some $M \in C_{\mathcal{F}}(N)$ with $|M| \geq 2$ (or, equivalently $i \in C$ for some $C \in \mathcal{C}(\mathcal{F})$ ). Thus, player $i$ has zero power if $i \notin \bigcup_{M \in C_{\mathcal{F}}(N),|M| \geq 2} M$. Throughout this paper we only consider positive power measures. For a union stable system $\mathcal{F}$ and a coalition $S \subseteq N$, we denote by $\mathcal{F}_{S}=\{T \in \mathcal{F}: T \subseteq S\}$ the union stable subsystem of those feasible coalitions for which all players belong to $S$. Note that $\mathcal{F}_{S}$ is union stable. We denote by $\mathcal{C}_{S}$ the supports with at least two players that only contains players of $S$.

In real life situations power or influence in union stable networks is of great importance. In modern societies large international firms have an important influence on society. As mentioned in the introduction, the network structure of people that belong to the boards of firms cannot be modelled by a simple graph, but it can be represented by a union stable system if we assume that communication or information can reach a board member of some company B from a board member of another company A, even when these two board members never meet in a common board, but are 'connected' to each other through other board members. The influence a board member has on society depends not only on his/her influence on the firms where he/she is a board member, but also on his/her influence on board members of other firms with whom he/she is 'connected'.

One of the best known power measures for simple graphs is the degree measure which assigns to every player in a communication graph its number of neighbours. This degree measure is generalized for union stable systems by Algaba et al. [1] who define the influence of a player $i$ by

$$
I_{i}(N, \mathcal{F})=\sum_{C \in \mathcal{C}_{i}} 1 /|C|,
$$

where $\mathcal{C}_{i}=\{C \in \mathcal{C}: i \in C\}$ is the collection of those basis coalitions that contain player $i$ and at least one other player. Note that in the example of board members, according to this influence measure the power of a member is less when he/she shares the board with more other people. In case the influence would not depend on the number of other members in a board, another generalization of the degree measure would just assign to every player the number of supports which he/she belongs to, i.e., $\bar{I}_{i}(N, \mathcal{F})=\left|\mathcal{C}_{i}\right|, i \in N$.

Given a positive power measure $\sigma$, we define the corresponding Harsanyi power solution $\varphi^{\sigma}: U S^{N} \rightarrow \mathbb{R}^{n}$ on the class of games on union stable systems 
by

$$
\varphi_{i}^{\sigma}(N, v, \mathcal{F})=\sum_{\substack{T \subseteq N, i \in T \\ \sum_{j \in T} \sigma_{j}\left(N, \mathcal{F}_{T}\right)>0}} \frac{\sigma_{i}\left(T, \mathcal{F}_{T}\right)}{\sum_{j \in T} \sigma_{j}\left(T, \mathcal{F}_{T}\right)} \Delta_{v^{\mathcal{F}}}(T) .
$$

Note that $\sum_{j \in T} \sigma_{j}\left(T, \mathcal{F}_{T}\right)=0$ if and only if $\mathcal{C}\left(\mathcal{F}_{T}\right)=\emptyset$, in which case $\Delta_{v^{\mathcal{F}}}(T)=0$.

In the following, for union stable system $\mathcal{F}$ and coalition $T$ such that $\sum_{j \in T} \sigma_{j}\left(N, \mathcal{F}_{T}\right) \neq 0$, we denote

$$
p_{i}^{\mathcal{F}, T}(\sigma)=\frac{\sigma_{i}\left(T, \mathcal{F}_{T}\right)}{\sum_{j \in T} \sigma_{j}\left(T, \mathcal{F}_{T}\right)}
$$

So, the Harsanyi power solution $\varphi^{\sigma}$ assigns to each union stable structure $(N, v, \mathcal{F})$ the Harsanyi solution (or sharing value) of the corresponding $\mathcal{F}$ restricted game $\left(N, v^{\mathcal{F}}\right)$ where any dividend $\Delta_{v^{\mathcal{F}}}(T)$ of coalition $T$ in the restricted game is distributed to the players in $T$ proportional to their powers in $\mathcal{F}_{T}$. Observe that the shares do not matter when all powers are zero, because that can only happen when all players of $T$ are dummy in $\mathcal{F}_{T}$ or the only feasible coalitions of $T$ are singletons, and thus the dividend of $T$ in $\left(N, v^{\mathcal{F}}\right)$ is equal to zero. Therefore, for notational convenience, in the following we take $p_{i}^{\mathcal{F}, T}(\sigma)=\frac{1}{\mid T}$, for all $i \in T$ whenever $\sum_{j \in T} \sigma_{j}\left(N, \mathcal{F}_{T}\right)=0$.

In this paper we will provide axiomatizations of the Harsanyi power solutions on the class $U S^{N}$ as well as on the subclass $U S I^{N}$. Before doing that we state the following lemma, generalizing a result of [9], establishing that dividends of nonfeasible coalitions are zero.

Lemma 1 Let $(N, v, \mathcal{F}) \in U S^{N}$ be a union stable structure. Then $\Delta_{v^{\mathcal{F}}}(S)=$ 0 for all $S \notin \mathcal{F}$.

Proof. Let $S \notin \mathcal{F}$. We prove the lemma by induction on the cardinality of $S$. If $|S|=1$, then $\Delta_{v^{\mathcal{F}}}(S)=v^{\mathcal{F}}(S)=0$, by zero-normality. Proceeding by induction suppose that $\Delta_{v^{\mathcal{F}}}(T)=0$ whenever $T \notin \mathcal{F}$ with $|T|<|S|$. Then,

$$
\begin{aligned}
\Delta_{v^{\mathcal{F}}}(S) & =v^{\mathcal{F}}(S)-\sum_{T \subset S} \Delta_{v^{\mathcal{F}}}(T) \\
& =\sum_{T \in C_{\mathcal{F}}(S)} v^{\mathcal{F}}(T)-\sum_{\substack{T \subset S \\
T \in \mathcal{F}}} \Delta_{v^{\mathcal{F}}}(T) \\
& =\sum_{T \in C_{\mathcal{F}}(S)} \sum_{\substack{H \subseteq T \\
H \in \mathcal{F}}} \Delta_{v^{\mathcal{F}}}(H)-\sum_{\substack{T \subset S \\
T \in \mathcal{F}}} \Delta_{v^{\mathcal{F}}}(T)=0 .
\end{aligned}
$$




\section{Properties}

First, we recall some standard axioms for allocation rules. An allocation rule $\gamma: U S^{N} \rightarrow \mathbb{R}^{N}$ is called component-efficient if for all $(N, v, \mathcal{F}) \in U S^{N}$ and $M \in C_{\mathcal{F}}(N)$, we have $\sum_{i \in M} \gamma_{i}(N, v, \mathcal{F})=v(M)$.

An allocation rule $\gamma: U S^{N} \rightarrow \mathbb{R}^{N}$ satisfies component dummy if for all $i \notin \bigcup_{M \in C_{\mathcal{F}}(N)} M$, we have $\gamma_{i}(N, v, \mathcal{F})=0$.

An allocation rule $\gamma: U S^{N} \rightarrow \mathbb{R}^{N}$ is additive if

$$
\gamma(N, v+w, \mathcal{F})=\gamma(N, v, \mathcal{F})+\gamma(N, w, \mathcal{F})
$$

for all $(N, v, \mathcal{F}),(N, w, \mathcal{F}) \in U S^{N}$.

Theorem 1 Let $\sigma$ be a positive power measure. The Harsanyi power solution $\varphi^{\sigma}: U S^{N} \rightarrow \mathbb{R}^{n}$ is an allocation rule that satisfies component efficiency, component-dummy and additivity.

Proof. Since $\Delta_{v^{\mathcal{F}}}(S)=0$ if there is no $T \in \mathcal{F}$ such that $S \subseteq T$, for every component $M \in C_{\mathcal{F}}(N)$ we have

$$
\begin{aligned}
\sum_{i \in M} \varphi_{i}^{\sigma}(N, v, \mathcal{F}) & =\sum_{i \in M} \sum_{\substack{T \subseteq N \\
i \in T}} p_{i}^{\mathcal{F}, T}(\sigma) \Delta_{v^{\mathcal{F}}}(T)=\sum_{T \subseteq M} \sum_{i \in T} p_{i}^{\mathcal{F}, T}(\sigma) \Delta_{v^{\mathcal{F}}}(T) \\
& =\sum_{T \subseteq M} \Delta_{v^{\mathcal{F}}}(T)=v^{\mathcal{F}}(M)=v(M)
\end{aligned}
$$

where the second equality follows from Lemma 1 and the fact that coalitions that contain players from different $\mathcal{F}$-components are not feasible, showing that $\varphi^{\sigma}$ satisfies component efficiency.

It is obvious that $\varphi^{\sigma}$ satisfies component dummy since $\Delta_{v^{\mathcal{F}}}(S)=0$ for all $S \subseteq N$ with $i \in S$ and $i \notin \bigcup_{M \in C_{\mathcal{F}}(N)} M$.

For all $v, w \in \mathcal{G}^{N}$ and all $\mathcal{F} \in U^{N},(v+w)^{\mathcal{F}}(S)=v^{\mathcal{F}}(S)+w^{\mathcal{F}}(S)$, and thus $\Delta_{(v+w)^{\mathcal{F}}}(S)=\Delta_{v^{\mathcal{F}}}(S)+\Delta_{w^{\mathcal{F}}}(S)$ for all $S \subseteq N$. Then

$$
\begin{aligned}
\varphi_{i}^{\sigma}(N, v+w, \mathcal{F}) & =\sum_{S \subseteq N, i \in S} p_{i}^{\mathcal{F}, S}(\sigma) \Delta_{(v+w)^{\mathcal{F}}}(S) \\
& =\sum_{S \subseteq N, i \in S} p_{i}^{\mathcal{F}, S}(\sigma)\left(\Delta_{v} \mathcal{F}(S)+\Delta_{w^{\mathcal{F}}}(S)\right) \\
& =\varphi_{i}^{\sigma}(N, v, \mathcal{F})+\varphi_{i}^{\sigma}(N, w, \mathcal{F}),
\end{aligned}
$$

showing that $\varphi^{\sigma}$ satisfies additivity.

Next, we generalize point unanimity as used in Algaba et al. [4] to axiomatize the Myerson value, in a similar way as the communication ability property for communication graph games of Borm et al. [6] is generalized in van den Brink et al. [9]. A union stable structure $(N, v, \mathcal{F})$ is called point 
anonymous if there exists a function $f:\{0,1, \ldots,|D(\mathcal{F})|\} \rightarrow \mathbb{R}$ such that $v^{\mathcal{F}}(S)=f(|S \cap D(\mathcal{F})|)$ for all $S \subseteq N$, where $D(\mathcal{F})=\left\{i \in N: \mathcal{C}_{i} \neq \emptyset\right\}$. When there is no confusion we will often write $D$ instead of $D(\mathcal{F})$. In [2] is shown that the Myerson value satisfies point anonymity ${ }^{2}$. However, in general Harsanyi power solutions do not satisfy this property.

Weakening point anonymity in a similar way as the corresponding property for communication graph games of [6] is weakened in [9], yields point unanimity. A union stable structure $(N, v, \mathcal{F})$ is called point unanimous if it is point anonymous with function $f:\{0,1, \ldots,|D|\} \rightarrow \mathbb{R}$ such that $f(k)=0$, for all $k \in\{0,1, \ldots,|D|-1\}$, i.e., the $\mathcal{F}$-restricted game is a multiple of the unanimity game on $D$. An allocation rule $\gamma$ satisfies point unanimity if all players that belong to at least one non-unitary support earn the same in any point unanimous union stable structure, while the other players earn zero. Using power measures for union stable systems this axiom can be generalized in a similar way as the communication ability property for communication graph games of [6] is generalized in [9] (using unanimous instead of anonymous games). So, taking into account the power or centrality of players in a union stable system, for a given power measure $\sigma$ we modify this axiom by requiring that the payoffs are allocated proportional to the players' power in the given union stable system.

$\sigma$-point unanimity. If $(N, v, \mathcal{F})$ is point unanimous, then there is $\alpha \in \mathbb{R}$ such that $f(N, v, \mathcal{F})=\alpha \sigma(N, \mathcal{F})$.

Observe that $\sigma$-point unanimity reduces to point unanimity when we take the equal power measure $\sigma=E$ given by

$$
E_{i}(N, \mathcal{F})= \begin{cases}1, & \text { if } \mathcal{C}_{i} \neq \emptyset \\ 0, & \text { if } \mathcal{C}_{i}=\emptyset\end{cases}
$$

Alternatively, any power measure can be used such as the influence measure $I$ or its alternative $\bar{I}$. Every Harsanyi power solution satisfies its corresponding $\sigma$-point unanimity.

Proposition 1 Let $\sigma$ be a positive power measure. The Harsanyi power solution $\varphi^{\sigma}$ satisfies $\sigma$-point unanimity on $U S^{N}$.

Proof. When $(N, v, \mathcal{F})$ is point unanimous, then $v^{\mathcal{F}}=v^{\mathcal{F}}(N) u_{D}$ and $\varphi^{\sigma}$ is obtained by distributing the unique nonzero dividend $\Delta_{v^{\mathcal{F}}}(N)=v^{\mathcal{F}}(N)$ among the players in $D$ according to the $\sigma$-measure, showing that $\varphi^{\sigma}$ satisfies $\sigma$-point unanimity.

\footnotetext{
${ }^{2}$ An allocation rule $\gamma$ satisfies point anonymity if all players that belong to at least one non-unitary support earn the same in any point anonymous union stable structure, while the other players earn zero.
} 


\section{Axiomatic characterizations on $U S I^{N}$}

Algaba et al. [4] axiomatized the Myerson value on the class $U S I^{N}$ by adding the superfluous player property to the properties of component efficiency, component-dummy, additivity and point unanimity, where the axioms are defined on the subclass $U S I^{N}$ similar as they are defined before on $U S^{N}$.

The support $H \in \mathcal{C}$ is called superfluous for $(N, v, \mathcal{F}) \in U S^{N}$ if $v^{\mathcal{C}}(\mathcal{A})=$ $v^{\mathcal{C}}(\mathcal{A} \backslash\{H\})$, for all $\mathcal{A} \subseteq \mathcal{C}$, i.e., if support $H$ is a null player in the conference game. An allocation rule $\gamma: U S^{N} \rightarrow \mathbb{R}^{N}$ has the superfluous support property if $\gamma(N, v, \mathcal{F})=\gamma(N, v, \overline{\mathcal{B} \backslash\{H\}})$, for all $(N, v, \mathcal{F}) \in U S^{N}$ and for every superfluous support $H \in \mathcal{C}$ for $(N, v, \mathcal{F})$.

It is satisfied that on the class $U S I^{N}$ all Harsanyi power solutions satisfy the superfluous support property.

Theorem 2 Let $\sigma$ be a positive power measure. The Harsanyi power solution $\varphi^{\sigma}$ satisfies the superfluous support property on $U S I^{N}$.

Proof. Since it is assumed that any game is zero-normalized, it follows from the definition of the $\mathcal{F}$-restricted game that, if $H$ is a superfluous support, then $v^{\mathcal{F}}=v^{\overline{\mathcal{B} \backslash\{H\}}}$, and thus

$$
\Delta_{v^{\mathcal{F}}}(S)=\Delta_{v^{\overline{\mathcal{B}} \backslash\{H\}}}(S), \quad S \subseteq N .
$$

When $H \nsubseteq \subseteq$, then $\mathcal{F}_{S}=(\overline{\mathcal{B} \backslash\{H\}})_{S}$ and so $\sigma_{i}\left(S, \mathcal{F}_{S}\right)=\sigma_{i}\left(S,(\overline{\mathcal{B} \backslash\{H\}})_{S}\right)$ for all $i \in S$, implying that the share of $i \in S$ in $\Delta_{v^{\mathcal{F}}}(S)$ is equal to the share of $i$ in $\Delta_{v \overline{\mathcal{B} \backslash\{H\}}}(S)$.

When $H \subseteq S$, then $S \notin \overline{\mathcal{B} \backslash\{H\}}$ (since otherwise, if $S$ can be written as a union of supports in $\mathcal{B} \backslash\{H\}$, then in $\mathcal{F}$ it can be written as the union of these supports and $H$ ), and thus $\Delta_{v_{\overline{\mathcal{B}} \backslash\{H\}}}(S)=0$ by Lemma 1. So, $\Delta_{v_{\overline{\mathcal{B}} \backslash\{H\}}}(S)=$ $\Delta_{v^{\mathcal{F}}}(S)=0$ and the shares don't matter. Hence

$$
\varphi^{\sigma}(N, v, \mathcal{F})=\varphi^{\sigma}(N, v, \overline{\mathcal{B} \backslash\{H\}})
$$

showing that $\varphi^{\sigma}$ satisfies the superfluous support property on $U S I^{N}$.

Note that in the proof above it is crucial that for any $(N, v, \mathcal{F}) \in U S I^{N}$ there cannot be a support $H$ such that also a proper subset of $H$ is a support in $\mathcal{F}^{3}$

\footnotetext{
${ }^{3}$ Consider, for example the union stable system $\mathcal{F}=\{\{1,2\},\{1,2,3\}\}$. Note that $(N, v, \mathcal{F}) \notin U S I^{N}$ since $\{1,2,3\}$ is a support and it can also be written as the union of the supports $\{1,2\}$ and $\{1,2,3\}$. Further, consider the unanimity game $v=u_{\{1,2,3\}}$. Then the influence measure on $N=\{1,2,3\}$ yields $I(N, \mathcal{F})=(5 / 6,5 / 6,1 / 3)$, so $\varphi^{I}(N, v, \mathcal{F})=$ $(5 / 6,5 / 6,1 / 3)$. Deleting from $\mathcal{F}$ the superfluous support $\{1,2\}$, we obtain the union stable system $\mathcal{F}^{\prime}=\{\{1,2,3\}\}$. Now the influence measure is given by $I\left(N, \mathcal{F}^{\prime}\right)=(1 / 3,1 / 3,1 / 3)$, so $\varphi^{I}\left(N, v, \mathcal{F}^{\prime}\right)=(1 / 3,1 / 3,1 / 3)$, showing that the outcome changed although we only deleted a superfluous support. Also, on $(N, v, \mathcal{F})$ the position value is not equal to $\varphi^{I}(N, v, \mathcal{F})$ since $\pi(N, v, \mathcal{F})=(1 / 3,1 / 3,1 / 3)$.
} 
Similar as shown for the Myerson value in [4] (i.e., for $\sigma=E$ ), it can be shown that on $U S I^{N}$ the above five axioms characterize the Harsanyi power solution that corresponds to the power measure that is applied to $\sigma$-point unanimity. Since the proof is similar for any Harsanyi power solution, we state this result without proof. ${ }^{4}$

Theorem 3 Let $\sigma$ be a positive power measure. The Harsanyi power solution $\varphi^{\sigma}: U S I^{N} \rightarrow \mathbb{R}^{n}$ is the unique allocation rule that satisfies component efficiency, component dummy, additivity, the superfluous support property and $\sigma$-point unanimity.

Besides weakening the communication ability property by using point unanimous games instead of point anonymous games, in [9] also the degree property, used in [6] to axiomatize the position value for communication graph games, is weakened in a similar way. The same we can do for the influence property, defined using the influence measure discussed before (see (2)) and used in [1] to characterize the position value for games on union stable systems. The triple $(N, v, \mathcal{F}) \in U S^{N}$ is called support anonymous if there exists a function $f:\{0,1, \ldots,|\mathcal{C}|\} \rightarrow \mathbb{R}$ such that $v^{\mathcal{C}}(\mathcal{A})=f(|\mathcal{A}|)$, for all $\mathcal{A} \subseteq \mathcal{C}$, i.e., if the conference game $\left(\mathcal{C}, v^{\mathcal{C}}\right)$ is anonymous. The triple $(N, v, \mathcal{F}) \in U S^{N}$ is called support unanimous if it is support anonymous with function $f$ satisfying $f(k)=0$, for $k \in\{0,1, \ldots,|\mathcal{C}|-1\}$, i.e., if the conference game $\left(\mathcal{C}, v^{\mathcal{C}}\right)$ is a multiple of the unanimity game on $\mathcal{C}$.

$\sigma$-influence property. If $(N, v, \mathcal{F})$ is support unanimous, then there is $\alpha \in \mathbb{R}$ such that $f(N, v, \mathcal{F})=\alpha \sigma(N, \mathcal{F})$.

Observe that the $\sigma$-influence property yields the (weak) influence property when we take $\sigma=I .^{5}$

The next lemma generalizes a result from [9] and states that $(i)$ on the class of all union stable structures support unanimity implies point unanimity, and (ii) on the subclass $U S I^{N}$ these properties are equivalent.

\section{Lemma 2}

(i) Let $(N, v, \mathcal{F}) \in U S^{N}$. If $(N, v, \mathcal{F})$ is support unanimous, then $(N, v, \mathcal{F})$ is also point unanimous.

(ii) Let $(N, v, \mathcal{F}) \in U S I^{N}$. Then $(N, v, \mathcal{F})$ is support unanimous if and only if $(N, v, \mathcal{F})$ is point unanimous.

\footnotetext{
${ }^{4}$ The proof can be obtained from the corresponding proof for the Myerson value in [4] by applying $\sigma$-point unanimity instead of point unanimity at every occasion where this is used.

${ }^{5}$ This weakens the influence property stating that for each $(N, v, \mathcal{F}) \in U S^{N}$ that is support anonymous, the payoffs to the players are proportional to their influence. This axiom is used in [1] to axiomatize the position value. This axiomatization still holds when the influence property is weakened by requiring it only for support unanimous union stable structures.
} 
Proof. (i) Let $(N, v, \mathcal{F}) \in U S^{N}$ be support unanimous. If $D=\emptyset$, then $\mathcal{C}=\emptyset$ implying that $v^{\mathcal{F}}(S)=0$, for all $S \subseteq N$ (by zero-normality of $v$ ), and thus $(N, v, \mathcal{F}) \in U S^{N}$ is point unanimous. Next, suppose that $D \neq \emptyset$. By definition of support unanimity, $v^{\mathcal{C}}=v^{\mathcal{F}}(N) u_{\mathcal{C}}$, and thus $v^{\overline{\mathcal{B} \backslash\{H\}}}(N)=$ $v^{\mathcal{C}}(\overline{\mathcal{B} \backslash\{H\}})=0$, for all $H \in \mathcal{C}$. For $T \subseteq N$, we distinguish two cases.

1. Let $T$ be such that $D \nsubseteq T$,

$$
v^{\mathcal{F}_{T}}(N)=\sum_{S \in C_{\mathcal{F}_{T}}(N)} v(S)=\sum_{S \in C_{\mathcal{F}_{T}}(T)} v(S)=v^{\mathcal{F}_{T}}(T)=v^{\mathcal{F}}(T),
$$

where the second equality follows from zero-monotonicity of $(N, v)$ and the fact that all players that do not belong to $T$ are dummies in $\left(N, \mathcal{F}_{T}\right)$. From $v^{\mathcal{C}}=v^{\mathcal{F}}(N) u_{\mathcal{C}}$, it further follows that $v^{\mathcal{F}_{T}}(N)=0$ since $\mathcal{F}_{T}$ is a proper subset of $\mathcal{F}$. Hence

$$
v^{\mathcal{F}}(T)=v^{\mathcal{F}_{T}}(N)=0 .
$$

2. Let $T$ be such that $D \subseteq T$, by definition of $v^{\mathcal{F}}$ and $C_{\mathcal{F}}(T)=C_{\mathcal{F}}(N)$,

$$
v^{\mathcal{F}}(T)=\sum_{S \in C_{\mathcal{F}}(T)} v(S)=\sum_{S \in C_{\mathcal{F}}(N)} v(S)=v^{\mathcal{F}}(N) .
$$

From these two cases we conclude that $v^{\mathcal{F}}=v^{\mathcal{F}}(N) u_{D}$, which proves $(i)$.

(ii) The 'only if' part follows from $(i)$. To prove the 'if' part, let $(N, v, \mathcal{F}) \in$ $U S I^{N}$ be point unanimous. Note that if we delete a support $H$ of $\mathcal{F}$ with $(N, v, \mathcal{F}) \in U S I^{N}$, then the set $D \notin \overline{\mathcal{B} \backslash\{H\}}$. Thus, if $v^{\mathcal{F}}=v^{\mathcal{F}}(N) u_{D}$, then $v^{\mathcal{C}}(\mathcal{C})=v^{\mathcal{F}}(N)$ and $v^{\mathcal{C}}(\mathcal{A})=v^{\overline{\mathcal{A}}}(N)=0$, for all $\mathcal{A} \subset \mathcal{C}$, implying that $v^{\mathcal{C}}=v^{\mathcal{F}}(N) u_{\mathcal{C}}$.

From this lemma it immediately follows that $\sigma$-point unanimity implies the $\sigma$-influence property whereas on the subclass $U S I^{N}, \sigma$-point unanimity and the $\sigma$-influence property are equivalent. As a corollary from Theorem 3 and Lemma 2.(ii) we immediately obtain the following result.

Corollary 1 Let $\sigma$ be a positive power measure. The Harsanyi power solution $\varphi^{\sigma}: U S I^{N} \rightarrow \mathbb{R}^{n}$ is the unique allocation rule that satisfies component efficiency, component-dummy, additivity, the superfluous support property and the $\sigma$-influence property.

For $\sigma=I$, Theorem 3 generalizes the axiomatization of the position value for communication graph games in [6], while for $\sigma=E$, this generalizes the axiomatization of the Myerson value being a corollary from [9].

Taking the influence measure, $\sigma=I$, it follows from [1] that on $U S I^{N}$, the position value is a Harsanyi power solution.

Corollary 2 If $(N, v, \mathcal{F}) \in U S I^{N}$ then $\varphi^{I}(N, v, \mathcal{F})=\pi(N, v, \mathcal{F})$. 
Corollary 2 shows that to define and compute the position value on $U S I^{N}$, we do not need the conference game, since it is a Harsanyi solution applied to the $\mathcal{F}$-restricted game $\left(N, v^{\mathcal{F}}\right)$. However, for arbitrary union stable systems the position value is not equal to $\varphi^{I}$ as is already shown in [9] for communication graph games. Clearly, since the influence measure is symmetric, it follows that $\varphi^{I}$ yields the Shapley value when there are no restrictions to the cooperation, i.e., if $\mathcal{F}=2^{N}$. On the other hand, it is well-known that the position value does not generalize the Shapley value in general, i.e., $\pi\left(v, 2^{N}\right)$ need not be equal to $S h(N, v)$.

As further corollaries we immediately obtain axiomatizations of the Myerson value and position value, of which some are already known.

Corollary 3 On the class of union stable cooperation structures $U S I^{N}$, it holds that:

(i) The position value $\pi: U S I^{N} \rightarrow \mathbb{R}^{n}$ is the unique allocation rule that satisfies component efficiency, component-dummy, additivity, the superfluous support property and I-point unanimity.

(ii) The Myerson value $\mu: U S I^{N} \rightarrow \mathbb{R}^{n}$ is the unique allocation rule that satisfies component efficiency, component-dummy, additivity, the superfluous support property and E-point unanimity.

(iii) The position value $\pi: U S I^{N} \rightarrow \mathbb{R}^{n}$ is the unique allocation rule that satisfies component efficiency, component-dummy, additivity, the superfluous support property and the I-influence property.

(iv) The Myerson value $\mu: U S I^{N} \rightarrow \mathbb{R}^{n}$ is the unique allocation rule that satisfies component efficiency, component-dummy, additivity, the superfluous support property and the E-influence property.

Part (iii) is already known from [1], while part (ii) is shown in [4]. Similarly as stated in [9] for cycle-free communication graph games, we may conclude that both the position value and the Myerson value on $U S I^{N}$ can be characterized by some influence property and some point unanimity property. Therefore, the difference between them is in the power measure that is used. For the position value this is the $I$-influence measure, whereas for the Myerson value it is the $E$-influence measure.

Let $U S U^{N} \subset U S^{N}$ be the class of support unanimous union stable structures. Next, we show that on this class the position value is equal to the Harsanyi power solution for the $I$-influence measure.

Proposition 2 Let $\sigma$ be a positive power measure. The Harsanyi power solution $\varphi^{\sigma}: U S U^{N} \rightarrow \mathbb{R}^{n}$ is the unique allocation rule that satisfies component efficiency, component dummy and the $\sigma$-influence property.

Proof. Let $(N, v, \mathcal{F}) \in U S U^{N}$ be a support unanimous union stable structure. If $D=\emptyset$, the payoffs are determined by component-dummy. Suppose that $D \neq \emptyset$. Then $\varphi^{\sigma}(N, v, \mathcal{F})=\alpha \sigma(N, \mathcal{F})$ by the $\sigma$-influence property. Let 
$\gamma(N, v, \mathcal{F})$ be another allocation rule satisfying component efficiency, component dummy and the $\sigma$-influence property. As $(N, v, \mathcal{F})$ is a support unanimous union stable structure, $\gamma(N, v, \mathcal{F})=\beta \sigma(N, \mathcal{F})$. In order to prove that $\beta=\alpha$, let $M \in C_{\mathcal{F}}(N)$.

Applying component-efficiency, it holds that $\sum_{i \in M} \gamma_{i}(N, v, \mathcal{F})=v(M)=$ $\sum_{i \in M} \varphi_{i}^{\sigma}(N, v, \mathcal{F})$. Therefore, $\sum_{i \in M} \beta \sigma_{i}(N, \mathcal{F})=\sum_{i \in M} \alpha \sigma_{i}(N, \mathcal{F})$. Hence, it is satisfied $(\beta-\alpha) \sum_{i \in M} \sigma_{i}(N, \mathcal{F})=0$. Since $\sum_{i \in M} \sigma_{i}(N, \mathcal{F}) \neq 0$ if $M \in$ $C_{\mathcal{F}}(N)$, we have that $\beta=\alpha$. Thus, $\gamma(N, v, \mathcal{F})=\varphi^{\sigma}(N, v, \mathcal{F})$.

Since the position value satisfies the $I$-influence property (see [1]), we obtain the following corollary.

Corollary 4 For every support unanimous union stable structure $(N, v, \mathcal{F})$, we have $\pi(N, v, \mathcal{F})=\varphi^{I}(N, v, \mathcal{F})$.

\section{Axiomatic characterizations on $U S^{N}$}

Since Harsanyi power solutions do not satisfy the superfluous support property on the class of all union stable structures, in order to provide an axiomatic characterization on the class of all union stable structures we will introduce other axioms and provide two axiomatizations. The first one generalizes the corresponding axiomatization of Harsanyi power solutions for communication graph games given in van den Brink et al. [9], the second one extends an axiomatization of the Myerson value for games on union stable systems studied in Algaba et al. [2].

An allocation rule $\gamma: U S^{N} \rightarrow \mathbb{R}^{N}$ satisfies the inessential support property if, for every union stable system $\mathcal{F}, c \in \mathbb{R}, T \in \mathcal{F}$ with $T \neq \varnothing$, and $H \in \mathcal{C}$ such that $H \nsubseteq T$, we have

$$
\gamma\left(N, c u_{T}, \mathcal{F}\right)=\gamma\left(N, c u_{T}, \overline{\mathcal{B} \backslash\{H\}}\right) .
$$

The inessential support property yields that given a unanimity game on a nonempty feasible coalition $T$, the solution does not depend on those supports which contain at least a player outside of coalition $T$. In other words, the essential supports for the unanimity games on a nonempty feasible coalition $T$ are those whose agents are all in $T$.

An allocation rule $\gamma: U S^{N} \rightarrow \mathbb{R}^{N}$ satisfies connectedness if $\gamma(N, v, \mathcal{F})=$ $\gamma(N, w, \mathcal{F})$ whenever $v^{\mathcal{F}}=w^{\mathcal{F}}$. Connectedness means that the solution only depends on the feasible coalitions.

Proposition 3 Let $\sigma$ be a positive power measure. The Harsanyi power solution $\varphi^{\sigma}: U S^{N} \rightarrow \mathbb{R}^{n}$ satisfies the inessential support property and connectedness.

Proof. In order to prove the inessential support property, let $v=c u_{T}, c \in \mathbb{R}$, with $T \in \mathcal{F}, T \neq \varnothing$ and $H \in \mathcal{C}$ such that $H \nsubseteq T$. Since $T \in \overline{\mathcal{B} \backslash\{H\}}$,

$$
\Delta_{v^{\mathcal{F}}}(T)=\Delta_{v^{\overline{\mathcal{B}} \backslash\{H\}}}(T)=c \text { and } \Delta_{v^{\mathcal{F}}}(S)=\Delta_{v^{\overline{\mathcal{B}} \backslash\{H\}}}(S)=0 \text {, for all } S \neq T \text {. }
$$


In addition to this, $\left(T, \mathcal{F}_{T}\right)=\left(T,(\overline{\mathcal{B} \backslash\{H\}})_{T}\right)$, and thus

$$
\sigma\left(T, \mathcal{F}_{T}\right)=\sigma\left(T,(\overline{\mathcal{B} \backslash\{H\}})_{T}\right)
$$

Therefore,

$$
\varphi^{\sigma}(N, v, \mathcal{F})=\varphi^{\sigma}(N, v, \overline{\mathcal{B} \backslash\{H\}}),
$$

showing that $\varphi^{\sigma}$ satisfies the inessential support property.

Connectedness holds straightforward since $\Delta_{v^{\mathcal{F}}}(S)=\Delta_{w^{\mathcal{F}}}(S)$, for all $S \subseteq$ $N$, whenever $v^{\mathcal{F}}=w^{\mathcal{F}}$.

Using these two properties we obtain the following axiomatic characterization of the Harsanyi power solutions on $U S^{N}$, generalizing the corresponding result of [9].

Theorem 4 Let $\sigma$ be a positive power measure. The Harsanyi power solution $\varphi^{\sigma}: U S^{N} \rightarrow \mathbb{R}^{n}$ is the unique allocation rule that satisfies component efficiency, component-dummy, additivity, $\sigma$-point unanimity, the inessential support property and connectedness.

Proof. It follows from Theorems 1 and Propositions 1 and 3 that $\varphi^{\sigma}$ is an allocation rule that satisfies the six properties on $U S^{N}$. To show uniqueness, let $(N, v, \mathcal{F}) \in U S^{N}$ and let $\gamma: U S^{N} \rightarrow \mathbb{R}^{n}$ be an allocation rule that satisfies the above properties.

To prove uniqueness, as $\gamma$ is additive and the game $(N, v)$ is zero-normalized, it is sufficient to show that for all $T \subseteq N$ with $|T| \geq 2$, and $\beta \in \mathbb{R}$, $\gamma\left(N, \beta u_{T}, \mathcal{F}\right)$ is uniquely determined. To prove this, fix $T \subseteq N$ with $|T| \geq 2$. We distinguish two cases:

1. Suppose that $T \in \mathcal{F}$. Then $\left(\beta u_{T}\right)^{\mathcal{F}}=\beta u_{T}$. From the inessential support property, $\gamma\left(N, \beta u_{T}, \mathcal{F}\right)=\gamma\left(N, \beta u_{T}, \mathcal{F}_{T}\right)$. Moreover, $\left(N, \beta u_{T}, \mathcal{F}_{T}\right)$ is a point unanimous union stable structure. So, $\sigma$-point unanimity yields that there exists $\alpha \in \mathbb{R}$ such that

$$
\gamma\left(N, \beta u_{T}, \mathcal{F}\right)=\gamma\left(N, \beta u_{T}, \mathcal{F}_{T}\right)=\alpha \sigma\left(N, \mathcal{F}_{T}\right) .
$$

Applying efficiency, it holds

$$
\sum_{i \in T} \gamma_{i}\left(N, \beta u_{T}, \mathcal{F}\right)=\sum_{i \in T} \alpha \sigma_{i}\left(N, \mathcal{F}_{T}\right)=\alpha \sum_{i \in T} \sigma_{i}\left(N, \mathcal{F}_{T}\right)=\beta
$$

and thus $\alpha=\frac{\beta}{\sum_{i \in T} \sigma_{i}\left(N_{,} \mathcal{F}_{T}\right)}$. We conclude that

$$
\gamma_{i}\left(N, \beta u_{T}, \mathcal{F}\right)= \begin{cases}\frac{\beta \sigma_{i}\left(N, \mathcal{F}_{T}\right)}{\sum_{i \in T} \sigma_{i}\left(N, \mathcal{F}_{T}\right)}, & \text { if } i \in T, \\ 0, & \text { otherwise, }\end{cases}
$$

and thus, $\gamma\left(N, \beta u_{T}, \mathcal{F}\right)=\varphi^{\sigma}\left(N, \beta u_{T}, \mathcal{F}\right)$. 
2. Suppose that $T \notin \mathcal{F}$. Let $\mathcal{S}=\{S \in \mathcal{F}: T \subset S\}$ be the collection of feasible subsets of $N$ that contain $T$, and let $c_{R} \in \mathbb{R}, R \in \mathcal{S}$, be such that,

$$
\left(\beta u_{T}\right)^{\mathcal{F}}=\sum_{R \in \mathcal{S}} c_{R} u_{R}
$$

(see van den Brink et al. [10] to determine $c_{R} \in \mathbb{R}$ and $R \in \mathcal{S}$ ). ${ }^{6}$ Applying Case 1 to each feasible coalition $R \in \mathcal{S}$, it is satisfied that

$$
\gamma\left(N, c_{R} u_{R}, \mathcal{F}\right)=\varphi^{\sigma}\left(N, c_{R} u_{R}, \mathcal{F}\right) .
$$

By additivity of $\gamma$ and $\varphi^{\sigma}$ it follows that $\gamma\left(N, \beta u_{T}, \mathcal{F}\right)=\varphi^{\sigma}\left(N, \beta u_{T}, \mathcal{F}\right)$. Again by additivity, $\gamma(N, v, \mathcal{F})=\varphi^{\sigma}(N, v, \mathcal{F})$.

As the Myerson value is the Harsanyi power solution for the equal power measure on $U S^{N}$, as a result we get a new characterization for the Myerson value on $U S^{N}$.

Corollary 5 The Myerson value $\mu: U S^{N} \rightarrow \mathbb{R}^{n}$ is the unique allocation rule that satisfies component efficiency, component-dummy, additivity, Epoint unanimity, the inessential support property and connectedness on $U S^{N}$.

Algaba et al. [2] provide an axiomatization of the Myerson value using the superfluous player property, generalizing a result for communication graph games in van den Nouweland [22]. A player $i \in N$ is called superfluous for $(N, v, \mathcal{F}) \in U S^{N}$ if $v^{\mathcal{F}}(S)=v^{\mathcal{F}}(S \backslash\{i\})$, for all $S \subseteq N$, i.e., it is a null player in the $\mathcal{F}$-restricted game $\left(N, v^{\mathcal{F}}\right)$. An allocation rule $\gamma$ satisfies the superfluous player property if for all $(N, v, \mathcal{F})$ and every player $i \in N$ that is superfluous for $(N, v, \mathcal{F})$ it holds $\gamma(N, v, \mathcal{F})=\gamma\left(N, v, \mathcal{F}_{N \backslash\{i\}}\right)$, where $\mathcal{F}_{N \backslash\{i\}}=\{F \in \mathcal{F}: F \subseteq N \backslash\{i\}\}$.

It can be shown in a similar way that every Harsanyi power solution satisfies this property.

Theorem 5 Let $\sigma$ be a positive power measure. The Harsanyi power solution $\varphi^{\sigma}: U S^{N} \rightarrow \mathbb{R}^{n}$ satisfies the superfluous player property.

Proof. Let $i \in N$ be a superfluous player in $(N, v, \mathcal{F})$. We have to prove $\varphi^{\sigma}(N, v, \mathcal{F})=\varphi^{\sigma}\left(N, v, \mathcal{F}_{N \backslash\{i\}}\right)$. Since $i$ is a null player in the game $\left(N, v^{\mathcal{F}}\right)$, $\varphi_{i}^{\sigma}(N, v, \mathcal{F})=0$. Since $i \notin \bigcup_{M \in C_{\mathcal{F}_{N \backslash\{i\}}(N)}} M$, by $\varphi^{\sigma}$ satisfying component dummy we have $\varphi_{i}^{\sigma}\left(N, v, \mathcal{F}_{N \backslash\{i\}}\right)=0$.

For the other players it is sufficient to show that $v^{\mathcal{F}}(S)=v^{\mathcal{F}_{N \backslash\{i\}}}(S)$, or equivalently that $v^{\mathcal{F}}(S \backslash\{i\})=v^{\mathcal{F}_{N \backslash\{i\}}}(S)$ for all $S \subseteq N$, whenever $i$ is a superfluous player in $(N, v, \mathcal{F})$. Since $C_{\mathcal{F}}(S \backslash\{i\})=C_{\mathcal{F}_{N \backslash\{i\}}}(S)$, we have

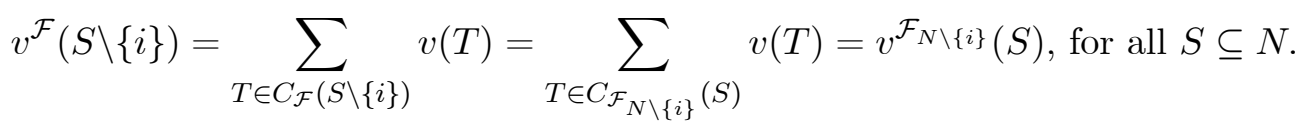

\footnotetext{
${ }^{6}$ Although [10] is about games on union closed systems, the proof is the same.
} 
In [2] (Theorem 3.7) the Myerson value is axiomatized by component efficiency, component dummy, additivity, the superfluous player property and point anonymity. Again, in the proof of uniqueness point anonymity is applied only to point unanimous games, and thus point anonymity can be weakened to point unanimity. But then, whenever point unanimity is applied we can as well apply $\sigma$-point unanimity, yielding the following theorem which we state without proof.

Theorem 6 Let $\sigma$ be a positive power measure. The Harsanyi power solution $\varphi^{\sigma}: U S^{N} \rightarrow \mathbb{R}^{n}$ is the unique allocation rule that satisfies component efficiency, component-dummy, additivity, the superfluous player property and $\sigma$-point unanimity.

Lemma 3.6 of [2] states that for every allocation rule that satisfies component efficiency, component-dummy, additivity and the superfluous player property, the payoff allocation to any game on a union stable system equals the payoff allocation assigned to the $\mathcal{F}$-restricted game on the same union stable system. But then it satisfies connectedness. So, replacing the inessential support property by the superfluous player property in Theorem 4, also we can do without connectedness.

\section{The Myerson value}

Next, we make some remarks on some special properties of the Myerson value as developed in [4]. First, although in [4] only point unanimous games are used, the Myerson value does satisfy point anonymity. However, in general, Harsanyi power solutions do not satisfy $\sigma$-point anonymity (stating that in a point anonymous game on union stable system the payoffs are allocated proportional to power measure $\sigma$ ), as already follows from [9] for communication graph games. Second, in [4] an axiomatization of the Myerson value by component efficiency, component-dummy, additivity, point unanimity and the strong superfluous support property is given. This last property states that the payoff allocation does not change after deleting a superfluous support. This property implies the superfluous support property, and therefore is not satisfied by all Harsanyi power solutions, since they already do not satisfy the superfluous support property on $U S^{N}$, in general.

We end this section with an example illustrating the solutions mentioned in this paper.

Example 1 Consider the player set $N=\{1,2,3,4\}$ and the union stable system given by $\mathcal{F}=\{\{1,2\},\{1,2,3\},\{2,3,4\}, N\}$. Let $v: 2^{N} \rightarrow \mathbb{R}$ be defined by $v=u_{\{1,2,3\}}$, for all $S \subseteq N$. Then,

$$
\mathcal{B}=\mathcal{C}=\{\{1,2\},\{1,2,3\},\{2,3,4\}\} .
$$

Notice that $v^{\mathcal{F}}=u_{\{1,2,3\}}$ and $(N, v, \mathcal{F}) \notin U S I^{N}$ since $N \in \mathcal{F}$ and it can be written as the union of the supports $\{1,2\} \cup\{2,3,4\}$ as well as the union 
of the supports $\{1,2,3\} \cup\{2,3,4\}$, i.e., the non-unitary feasible coalition $N$ does not admit a unique expression as a union of non-unitary supports. Note that the second condition which defines the subclass of union stable structures $U S I^{N}$ is not satisfied either since $\{1,2,3\} \cap\{2,3,4\}=\{2,3\} \notin \mathcal{F}$. Computing the Myerson value, the position value, the Harsanyi power solution obtained for the E-influence measure and the Harsanyi power solution obtained for the I-influence measure, we obtain

$$
\begin{aligned}
\mu(N, v, \mathcal{F}) & =\varphi^{E}(N, v, \mathcal{F})=\left(\frac{1}{3}, \frac{1}{3}, \frac{1}{3}, 0\right) . \\
\pi(N, v, \mathcal{F}) & =\left(\frac{11}{36}, \frac{13}{36}, \frac{10}{36}, \frac{2}{36}\right) . \\
\varphi^{I}(N, v, \mathcal{F}) & =\left(\frac{5}{12}, \frac{5}{12}, \frac{2}{12}, 0\right) .
\end{aligned}
$$

\section{References}

[1] Algaba E, Bilbao JM, Borm P, López JJ (2000) The position value for union stable systems. Math Meth Oper Res 52: 221-236

[2] Algaba E, Bilbao JM, Borm P, López JJ (2001) The Myerson value for union stable structures. Math Meth Oper Res 54: 359-371

[3] Algaba E, Bilbao JM, van den Brink, R, Jiménez-Losada A (2004) Cooperative games on antimatroids. Discrete Math 282: 1-15

[4] Algaba E, Bilbao JM, van den Brink, R, López JJ (2011) The Myerson value and superfluous supports in union stable systems. Tinbergen Discussion Paper TI 11/127, Tinbergen Institute, VU University, Amsterdam.

[5] Bilbao JM (2003) Cooperative games on augmenting systems. SIAM J. Discrete Math. 17: 122-133

[6] Borm P, Owen G, Tijs SH (1992) On the position value for communication situations. SIAM J. Discrete Math. 5:305-320

[7] Brink R van den (1997) An axiomatization of the disjunctive permission value for games with a permission structure. Int J Game Theory 26: $27-43$

[8] Brink R van den (2011) On hierarchies and communication. forthcoming in Soc Choice and Welf, DOI: 10.1007/s00355-011-0557-y

[9] Brink R van den, van der Laan G, Pruzhansky V (2011) Harsanyi power solutions for graph-restricted games. Int J Game Theory 40: 87-110

[10] Brink R van den, Katsev I, van der Laan G (2011) Axiomatizations of two types of Shapley values for games on union closed systems. Economic Theory 47: 175-188

[11] Conyon, MJ, Muldoon MR (2006) The Small World of Corporate Boards. Journal of Business Finance and Accounting 33: 1321-1343

[12] Derks J, Haller H, Peters H (2000) The selectope for cooperative TUgames. Int J Game Theory 29: 23-38 
[13] Derks J, van der Laan G, Vasil'ev (2010) On the Harsanyi payoff vectors and Harsanyi imputations. Theory Dec 68: 301-310

[14] Gilles RP, Owen G, Brink R van den (1992) Games with permission structures: the conjunctive approach. Int J Game Theory 20: 277-293

[15] Faigle U, Kern W. (1992) The Shapley value for cooperative games under precedence constraints. Int J Game Theory 21: 249-266

[16] Faigle U, Grabish M, Heyne M (2010) Monge extensions of cooperation and communication structures. European J. of Operational Research 206: $104-110$

[17] Harsanyi JC (1959) A bargaining model for cooperative $n$-person games. In: Tucker AW, Luce RD (eds) Contributions to the theory of games IV. Princenton University Press, Princenton, pp 325-355

[18] Meessen R (1988) Communication games. Master's thesis, Dept of Mathematics, University of Nijmegen, the Netherlands. (In Dutch)

[19] Mizruchi MS, Bunting D (1981) Influence in Corporate Networks: An Examination of Four Measures. Administrative Science Quarterly 26: 475-489.

[20] Myerson RB (1977) Graphs and cooperation in games. Math. Oper. Res. 2: $225-229$

[21] Myerson RB (1980) Conference structures and fair allocation rules. Int. J. Game Theory 9: 169-182

[22] Nouweland A van den (1993) Games and graphs in economics situations. PhD Dissertation, Tilburg University, The Netherlands

[23] Nouweland A van den, Borm P, Tijs SH (1992) Allocation rules for hypergraph communication situations. Int J Game Theory 20: 255-268

[24] Owen G (1986) Values of graph-restricted games. SIAM J on Algebraic and Discrete Methods 7: 210-220

[25] Potters JAM, Reijnierse H (1995) $\Gamma$-Component additive games. Int J Game Theory 24: 49-56

[26] Shapley LS (1953) A value for n-person games. In: Kuhn HW, Tucker AW (eds.). Contributions to the Theory of Games, Vol II. Princeton UP, pp. 307-317

[27] Vasil'ev VA (1982) Support function on the core of a convex game. Optimizacija 21: 30-35 (in Russian)

[28] Vasil'ev VA (2003) Extreme points of the Weber polytope. Discretnyi Analiz i Issledonaviye Operatsyi, Ser 1 10: 17-55 (in Russian) 